\title{
The fast light of CsI(Na) crystals ${ }^{*}$
}

\author{
Xilei Sun ${ }^{1 ; 1)}$ Junguang $\mathrm{Lu}^{1} \quad \mathrm{Tao} \mathrm{Hu}^{1}$ Li Zhou $^{1} \quad$ Jun Cao $^{1} \quad$ Yifang Wang $^{1} \quad$ Liang Zhan $^{1}$ Boxiang Yu ${ }^{1}$ \\ Xiao Cai $^{1}$ Jian Fang ${ }^{1} \quad$ Yuguang Xie $^{1}$ Zhenghua An ${ }^{1}$ Zhigang Wang ${ }^{1}$ Zhen Xue ${ }^{1}$ Aiwu Zhang ${ }^{1}$ \\ Qiwen $\mathrm{Lu}^{2}$ Feipeng Ning ${ }^{1} \quad$ Yongshuai $\mathrm{Ge}^{1} \quad$ Yingbiao Liu ${ }^{1}$ \\ ${ }^{1}$ Institute of High Energy Physics, CAS, Beijing 100049, China \\ ${ }^{2}$ Shanxi University, Taiyuan 030006, China
}

\begin{abstract}
The responds of different common alkali halide crystals to alpha-rays and gamma-rays are tested in this research. It is found that only $\mathrm{CsI}(\mathrm{Na})$ crystals have significantly different waveforms between alpha and gamma scintillations, while others have not this phenomena. It is suggested that the fast light of $\mathrm{CsI}(\mathrm{Na})$ crystals arises from the recombination of free electrons with self-trapped holes of the host crystal CsI. Self-absorption limits the emission of fast light of $\mathrm{CsI}(\mathrm{Tl})$ and $\mathrm{NaI}(\mathrm{Tl})$ crystals.
\end{abstract}

Key words CsI(Na), Waveform, Ionization, Scintillation, Exciton, Self-absorption

PACS 29.40.Mc

\section{Introduction}

CsI(Na) crystals are important scintillators for electromagnetic calorimetry in experimental particle physics. According to catalogues of main scintillation crystals producers (Saint-Gobain, AMCRYS-H et al.), the decay time of $\gamma$-scintillations in $\operatorname{CsI}(\mathrm{Na})$ amounts to approximately $630 \mathrm{~ns}$ with the peak wavelength of $420 \mathrm{~nm}$. In fact, our previous paper reveals that there are fast components hiding in the slow $\gamma$-scintillations of $\operatorname{CsI}(\mathrm{Na})$ crystals, and the proportion of that is larger responding to higher $\mathrm{dE} / \mathrm{dx}$ particles [1].

In this paper, we report the result of the testing of other common alkali halide crystals responding to alpha-rays and gamma-rays. The luminescence process of common alkali halide crystals is discussed in detail at the end of this paper.

\section{Experimental set-up}

The schematic of the experimental set-up is illustrated in Fig. 1. Two R8778 PMTs are directly attached to the top and the bottom surfaces of the crystal. The PMT signals are sent to the oscilloscope and discriminators (NIM CAEN N840) via a fan-out module (NIM CAEN N625). The discriminator thresholds are set to be equivalent to 0.5 single photoelectrons, which are calibrated by a pulsed LED light source. The noises of PMT can then be effectively suppressed to $0.055 \mathrm{~Hz}$, which is mainly caused by Cherenkov light, by the coincidence of the two discriminator signals with a width of $200 \mathrm{~ns}$. The oscilloscope is a TDS3054C with a sampling frequency of $5 \mathrm{GS} / \mathrm{s}$ and a memory depth of $2 \mu \mathrm{s}$ (10000 points, $0.2 \mathrm{~ns} /$ channel) for each of 4 channels. The high-speed sampling rate is a necessary condition for fast waveform analysis.

\footnotetext{
${ }^{*}$ Supported by Technological Innovation Project of Institute of High Energy Physics

${ }^{1)}$ E-mail: sunxl@ihep.ac.cn
} 
The crystals tested in this study are $\mathrm{CsI}(\mathrm{Na})$, CsI(Tl), NaI(Tl) and pure CsI. The detail properties of them are listed in Table 1 .
Gamma-rays from a 0.5 micro-Curie ${ }^{137} \mathrm{Cs}$ source and alpha-rays from a 5 micro-Curie ${ }^{239} \mathrm{Pu}$ source are used to excite the crystals.

Table. 1 The crystals tested in the research.

\begin{tabular}{|c|c|c|c|c|}
\hline Crystals & $\mathrm{CsI}(\mathrm{Na})$ & $\mathrm{CsI}(\mathrm{Tl})$ & $\mathrm{NaI}(\mathrm{Tl})$ & $\mathrm{CsI}$ \\
\hline Doping & $\mathrm{Na}$ & $\mathrm{Tl}$ & $\mathrm{Tl}$ & - \\
\hline Mole$\%$ & $\sim 0.02$ & $\sim 0.2$ & $\sim 0.2$ & - \\
\hline Size $(\mathrm{cm})$ & $2.5 \times 2.5 \times 2.5$ & $\Phi 2.5 \times 2.5$ & $2 \times 2 \times 2$ & $\Phi 2.5 \times 2.5$ \\
\hline
\end{tabular}

the rise time is $\sim 5 \mathrm{~ns}$. The decay time of slow

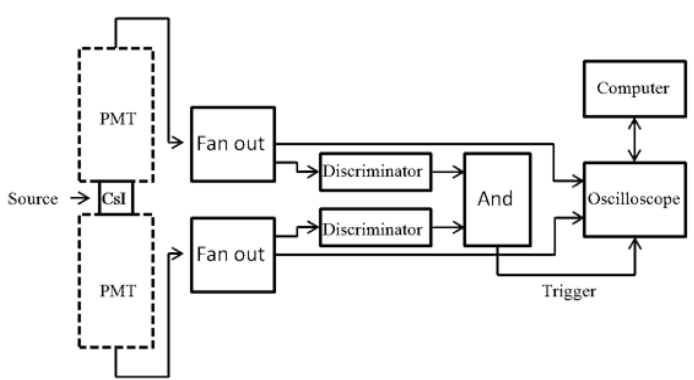

Fig. 1. Experimental set-up for the test of the crystals.

\section{Results and discussion}

The measured waveform profile histograms of $\gamma$-scintillations and $\alpha$-scintillations from different crystals are shown respectively below. Here we chose waveforms of full energy deposition to build the histograms, which are $661.7 \mathrm{keV}$ for gamma and 5.2 MeV for alpha. The values of the rise time are read from the amplification plots of the leading edge directly, and that of decay time are get from an exponential fitting of the trailing edge.

\subsection{Waveforms of CsI(Na)}

Waveform profile histograms of $\gamma$-scintillations $(661.7 \mathrm{keV})$ and $\alpha$-scintillations (5.2 MeV) from $\mathrm{CsI}(\mathrm{Na})$ crystals are shown in Fig. 2. The waveforms of $\gamma$-scintillations have a rise time of $\sim 40 \mathrm{~ns}$ and a decay time of $\sim 670 \mathrm{~ns}$. In comparison, the waveforms of $\alpha$-scintillations with old or new surface have significant fast components. The decay time is only $\sim 17 \mathrm{~ns}$ and components of $\alpha$-scintillations is shorter than that of $\gamma$-scintillations, which is $\sim 490 \mathrm{~ns}$.

Here, the old surface is the surface layer of $\mathrm{CsI}(\mathrm{Na})$ crystals with sodium ions losing caused by the moisture absorption of sodium; the new surface is that of grinding off the old layer. Thallium-doped crystals do not have this phenomenon.

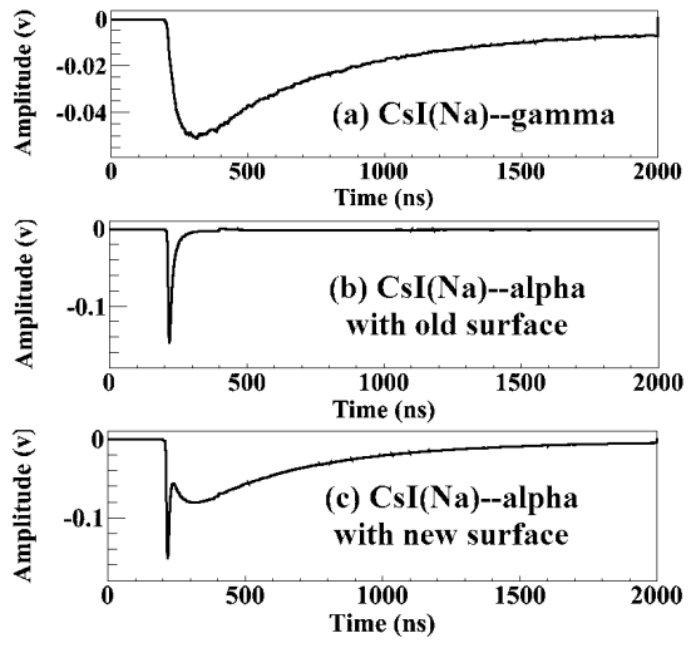

Fig. 2 Waveform profile histograms from $\mathrm{CsI}(\mathrm{Na})$ crystals. (a) $\gamma$-scintillations of $661.7 \mathrm{keV}$ (b) $\alpha$-scintillations of $5.2 \mathrm{MeV}$ with old surface (c) $\alpha$-scintillations of $5.2 \mathrm{MeV}$ with new surface.

\subsection{Waveforms of CsI(TI)}

Waveform profile histograms of $\gamma$-scintillations $(661.7 \mathrm{keV})$ and $\alpha$-scintillations (5.2 MeV) from CsI(Tl) crystals are shown in Fig. 3 . The rise time of $\alpha$-scintillations is $\sim 35 \mathrm{~ns}$ and the decay time is $\sim 670 \mathrm{~ns}$. There is not fast light. Similar, the waveforms of $\gamma$-scintillations have a rise time $\sim 50 \mathrm{~ns}$ and a longer decay time $\sim 1080$ ns. Although the different decay time can be 
used for alpha-gamma separation, but the performance is much less than $\operatorname{CsI}(\mathrm{Na})$ crystals by using the fast light.
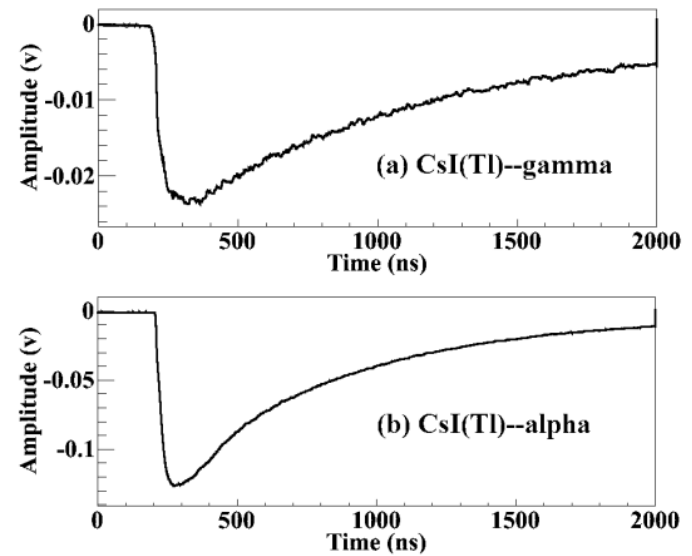

Fig. 3 Waveform profile histograms from $\mathrm{CsI}(\mathrm{Tl})$ crystals. (a) $\gamma$-scintillations of $661.7 \mathrm{keV}$ (b) $\alpha$-scintillations of $5.2 \mathrm{MeV}$.

\subsection{Waveforms of $\mathrm{NaI}(\mathrm{Tl})$}

Waveform profile histograms of $\gamma$-scintillations $(661.7 \mathrm{keV})$ and $\alpha$-scintillations (5.2 MeV) from $\mathrm{NaI}(\mathrm{Tl})$ crystals are shown in Fig. 4. The rise time of $\alpha$-scintillations is $\sim 10 \mathrm{~ns}$ and the decay time is $\sim 170 \mathrm{~ns}$. Similar, the waveforms of $\gamma$-scintillations have a rise time $20 \mathrm{~ns}$ and a slightly longer decay time $\sim 250 \mathrm{~ns}$. It is difficult to separate alpha and gamma by the decay time.
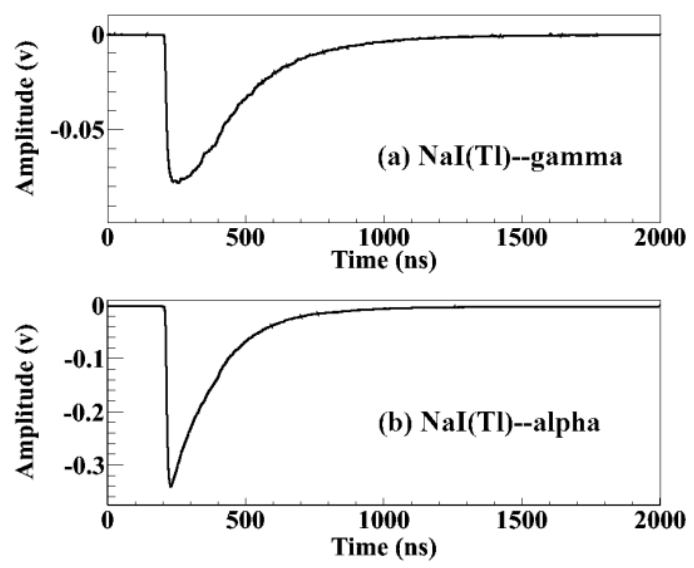

Fig. 4 Waveform profile histograms from $\mathrm{NaI}(\mathrm{Tl})$ crystals. (a) $\gamma$-scintillations of $661.7 \mathrm{keV}$ (b) $\alpha$-scintillations of $5.2 \mathrm{MeV}$.

\subsection{Waveforms of pure CsI}

Finally, waveform profile histograms of $\gamma$-scintillations $(661.7 \mathrm{keV})$ and $\alpha$-scintillations (5.2 MeV) from pure CsI crystals are shown in Fig. 5. The rise time of $\alpha$-scintillations is $\sim 5 \mathrm{~ns}$ and the decay time is $\sim 15 \mathrm{~ns}$. Similar, the waveforms of $\gamma$-scintillations have a rise time $\sim 5$ ns and a decay time $\sim 22$ ns.
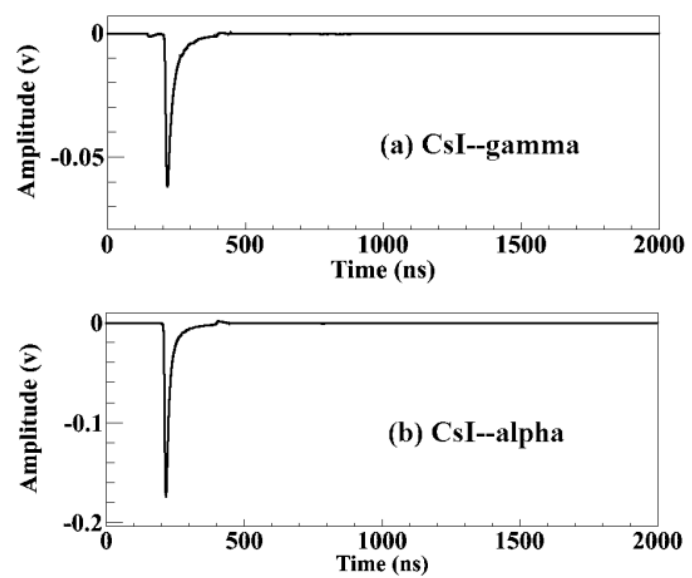

Fig. 5 Waveform profile histograms from pure CsI crystals. (a) $\gamma$-scintillations of $661.7 \mathrm{keV}$ (b) $\alpha$-scintillations of $5.2 \mathrm{MeV}$.

The summary of the measured waveform parameters are listed in Table 2. The present measurements of waveforms lead to two facts: (1) The fast light of $\mathrm{CsI}(\mathrm{Na})$ crystals can be significantly excited by alpha-rays, while which cannot be excited by gamma-rays. (2) The luminescence of gamma and alpha from the other common alkali halide crystals is not significantly different. It, thus, appears from these two facts that there are different aspects of the luminescence mechanisms between $\mathrm{CsI}(\mathrm{Na})$ crystals and $\mathrm{CsI}(\mathrm{Tl})$ or $\mathrm{NaI}(\mathrm{Tl})$ crystals. 
Table. 2 Summary of waveform parameters.

\begin{tabular}{|c|c|c|c|c|c|c|c|c|}
\hline Crystals & \multicolumn{2}{|c|}{$\operatorname{CsI}(\mathrm{Na})$} & \multicolumn{2}{c|}{$\operatorname{CsI}(\mathrm{Tl})$} & \multicolumn{2}{c|}{$\mathrm{NaI}(\mathrm{Tl})$} & \multicolumn{2}{c|}{ CsI } \\
\hline Source & $\gamma$ & $\alpha$ & $\gamma$ & $\alpha$ & $\gamma$ & $\alpha$ & $\gamma$ & $\alpha$ \\
\hline Rise time (ns) & 40 & 5 & 50 & 35 & 20 & 10 & 5 & 5 \\
\hline Decay time (ns) & 670 & $17_{\text {fast }}$ & 1080 & 670 & 250 & 170 & 22 & 15 \\
\cline { 2 - 7 } & & $490_{\text {slow }}$ & & & & & & \\
\hline
\end{tabular}

\subsection{Discussion}

The scintillation process in common alkali halide crystals has been discussed by Stephen E. Derenzo and Marvin J. Weber in reference [2], which can be described in several steps:

\section{i. Ionization}

Ionization occurs when the particles injected into the crystals, and the average energy needed to produce an electron-hole pair is about $20 \mathrm{eV}$ for CsI and NaI crystals.

\section{ii. Relaxation of Electrons and Holes}

The electrons and the holes resulting from the ionization process form separated charge carriers that cause the surrounding atoms to rearrange themselves. In CsI and $\mathrm{NaI}$ crystals, the self-trapped hole takes the form of a so-called $V_{k}$ center, where two Iodine atoms share the hole by pulling together and forming a covalent bond.

iii. Carrier Diffusion

In CsI and $\mathrm{NaI}$ crystals, the valance bands are filled and an excess electron is spatially diffuse in an essentially empty conduction band. On the other hand, holes are generally localized and will only diffuse if thermal vibrations are able to move the hole from one trapping site to another.

\section{iv. Formation of different excitons}

A diffuse electron may be trapped by an activator atom and then a self-trapped hole also be captured by it through thermal migration. The result is an activator-trapped-exciton. A diffuse electron may also be trapped immediately by a self-trapped hole resulting a self-trapped-exciton. The probability that a given electron will recombine with a self-trapped hole rather than suffer capture at activator atom site will be an increasing function of the density of self-trapped holes, hence, an increasing function of ionization density, while a decreasing function of concentration of activator atoms.

\section{v. Radiative Emission}

The de-excitation of two different kinds of excitons results two different scintillations. Thermal quenching is a common non-radiative process and occurs if thermal vibrations can deform exciton to ground state. Self-absorption is another constraint of scintillations if the emission band overlaps with the absorption band.

Based on the above process, it is noted that the fast scintillations with decay time of about $20 \mathrm{~ns}$ and wavelength of about $310 \mathrm{~nm}$ from pure CsI or $\mathrm{NaI}$ crystals are dominated by self-trapped-excitons for both gamma and alpha particles. The fast light of $\alpha$-scintillations from $\mathrm{CsI}(\mathrm{Na}) \quad$ crystals arises from self-trapped-excitons, while slow components of both alpha and gamma scintillations are dominated by activator-trapped-excitons. Fortunately, the absorption bands of $\mathrm{CsI}(\mathrm{Na})$ are all below $300 \mathrm{~nm}$ [3], thus, the fast light can be propagation through crystals.

$\mathrm{CsI}(\mathrm{Tl})$ and $\mathrm{NaI}(\mathrm{Tl})$ crystals have the same mechanism of scintillation, while, a discrepancy is that the emission bands of fast light overlaps with the absorption bands [4] [5], hence, the fast light of high ionization density particles cannot be significantly observed. UV self-absorption of $\mathrm{CsI}(\mathrm{Tl})$ and $\mathrm{NaI}(\mathrm{Tl})$ crystals may be due to the presence of $\mathrm{Tl}$ atom, which can be excited by the UV light.

The transmittance of different crystals measured by a DH-2000-BAL Deuterium-Halogen Light Source and a QE65000 Scientific-grade Spectrometer is 
shown in Fig. 6. It can clearly be seen that the light of wavelength less than $320 \mathrm{~nm}$ is strongly absorbed for CsI(Tl) crystals. The decay length of $310 \mathrm{~nm}$ light is $\sim 33 \mathrm{~cm}$ for $\mathrm{CsI}(\mathrm{Na})$ crystals according to the measurements.

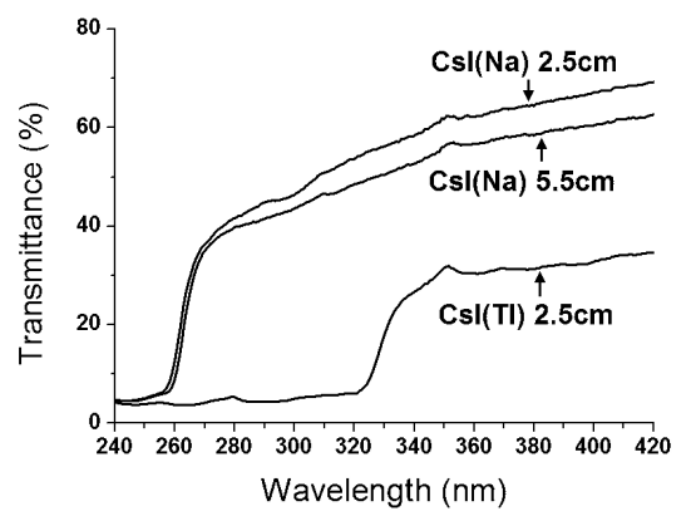

Fig. 6 Transmittance of different crystals.

\section{Conclusions}

Different alkali halide crystals are tested, the fast components of $\alpha$-scintillations only can be significantly found from $\operatorname{CsI}(\mathrm{Na})$ crystals. Self-absorption limits the emission of fast light from $\mathrm{CsI}(\mathrm{Tl})$ and $\mathrm{NaI}(\mathrm{Tl})$ crystals. Hence, $\mathrm{CsI}(\mathrm{Na})$ is the unique crystals among common alkali halide crystals, which can be used to doing particle identification by different kinds of luminescence mechanisms.

The fast light of $\mathrm{CsI}(\mathrm{Na})$ crystals can be significantly excited by particles with high ionization density like alphas rather than electrons. As a result, the high $\mathrm{dE} / \mathrm{dx}$ particles can be easily distinguished from gamma-rays and electrons. Therefore, $\mathrm{CsI}(\mathrm{Na})$ crystals are suitable materials for neutron detection and dark matter detection.

\section{Acknowledgments}

We are thankful to Liangjian Wen for his support.

\section{References}

[1] arXiv:1102.2727v1 [physics.ins-det]

[2] Stephen E. Derenzo and Marvin J. Weber. Nucl. Instr. and Meth. A, 1999, 422: 111-118

[3] Olive Lee Hsu. Phys. Rev. B, 1977, 15: 5821-5833

[4] R. Gwin and R. B. Murray. Phys. Rev, 1963, 131: $508-512$

[5] Eiichi Matsui. J. Phys. Soc. Jpn, 1967, 22: 819-830 